\title{
Application of an Energy-Vorticity Turbulence Model to Fully Rough Pipe Flow
}

\author{
E. B. Fowler, ${ }^{*}$ D. F. Hunsaker ${ }^{\dagger}$ and W. F. Phillips, ${ }^{\ddagger}$ \\ Utah State University, Logan, Utah 84322-4130
}

\begin{abstract}
Based on a more direct analogy between turbulent and molecular transport, a foundation was recently presented for an energy-vorticity turbulence model. The new turbulent-energytransport equation contains two closure coefficients; a viscous-dissipation coefficient and a turbulent-transport coefficient. To help evaluate the closure coefficients and provide insight into the energy-vorticity turbulence variables, fully rough pipe flow is considered. For this fully developed flow, excellent agreement with experimental data for velocity profiles and friction factors is attained over a wide range of closure coefficients, provided that a given relation between the coefficients is maintained.
\end{abstract}

\section{Nomenclature}

$A_{\lambda 0}=$ empirical coefficient, Eqs. (33) and (36)

$A_{\lambda 1} \quad=$ empirical coefficient, Eqs. (33) and (49)

$A_{\lambda 10}=$ empirical coefficient, Eqs. (37) and (49)

$A_{\lambda 11}=$ empirical coefficient, Eqs. (38) and (49)

$A_{\lambda 12}=$ empirical coefficient, Eqs. (39) and (49)

$A_{\lambda 13}=$ empirical coefficient, Eqs. (40) and (49)

$B_{\lambda 0}=$ empirical coefficient, Eqs. (33) and (50)

$B_{\lambda 00}=$ empirical coefficient, Eqs. (41) and (50)

$B_{\lambda 01}=$ empirical coefficient, Eqs. (42) and (50)

$B_{\lambda 02}=$ empirical coefficient, Eqs. (43) and (50)

$B_{\lambda 03}=$ empirical coefficient, Eqs. (44) and (50)

$B_{\lambda 1} \quad=$ empirical coefficient, Eqs. (33) and (51)

$B_{\lambda 1 \mathrm{C}}=$ empirical coefficient, Eqs. (45) and (51)

$B_{\lambda 11}=$ empirical coefficient, Eqs. (46) and (51)

$B_{\lambda 2}=$ empirical coefficient, Eqs. (33) and (52)

$B_{\lambda 2 \mathrm{C}}=$ empirical coefficient, Eqs. (47) and (52)

$B_{\lambda 21}=$ empirical coefficient, Eqs. (48) and (52)

$C_{f}=$ Fanning friction factor also called the skin-friction coefficient

$C_{0-7}=$ empirical coefficients, Eqs. (29) and (30)

$C_{0-4}=$ empirical coefficients, Eqs. (39), (43), and (47)

$C_{\lambda}=$ turbulence model closure coefficient, Eq. (20)

$C_{\mu} \quad=$ turbulence model closure coefficient, Eq. (3)

${ }^{*} \mathrm{PhD}$ Candidate, Mechanical and Aerospace Engineering Department, 4130 Old Main Hill.

${ }^{\dagger}$ PhD Candidate, Mechanical and Aerospace Engineering Department, 4130 Old Main Hill; currently Design Engineer, Scaled Composites, 1624 Flight Line, Mojave, CA 93501-1663.

*Professor, Mechanical and Aerospace Engineering Department, 4130 Old Main Hill. Senior Member AIAA. 


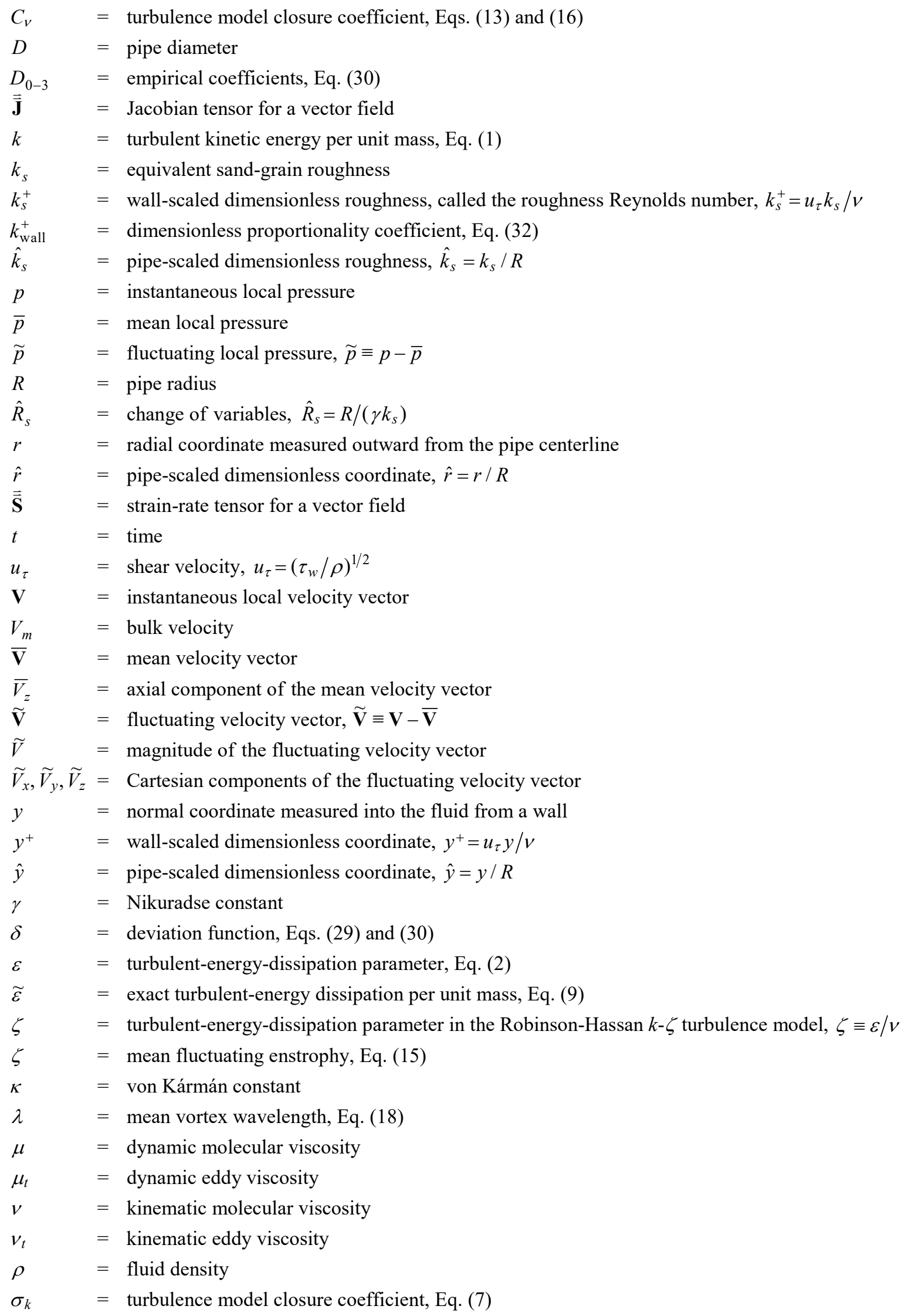




$\begin{array}{ll}\overrightarrow{\bar{\tau}} & =\text { turbulent Reynolds stress tensor, Eq. (4) } \\ \tau_{w} & =\text { pipe wall shear stress } \\ \omega & =\text { turbulent-energy-dissipation frequency, } \omega \equiv \varepsilon /\left(C_{\mu} k\right) \\ \omega^{+} & =\text {wall-scaled dimensionless dissipation frequency, } \omega^{+}=v \omega / u_{\tau}^{2} \\ \widetilde{\omega} & =\text { root-mean-square fluctuating vorticity, Eq. (10) }\end{array}$

\section{Introduction}

Wany of the turbulence models that are now commonly used for computational fluid dynamics (CFD) are based

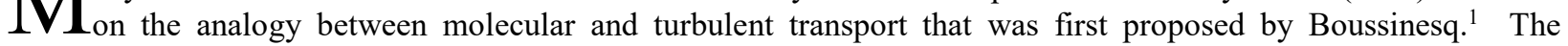
majority of these turbulence models are usually classified as either $k-\varepsilon, k-\omega$, or $k-\zeta$ models. Conventional $k-\varepsilon, k-\omega$, and $k-\zeta$ turbulence models are often thought of as being fundamentally different. Yet, in a larger sense, these three model classifications could all be thought of as energy-dissipation models. This is because all such models are based on the hypothesis that Boussinesq's eddy viscosity is proportional to the product of the root-mean-square (RMS) fluctuating velocity, or $k^{1 / 2}$, and the dissipation length scale $k^{3 / 2} / \varepsilon$. The parameters $k$ and $\varepsilon$ are defined in terms of the fluctuating velocity as

$$
\begin{gathered}
k \equiv \frac{1}{2} \overline{\tilde{\mathbf{V}} \cdot \widetilde{\mathbf{V}}}=\frac{1}{2} \overline{\widetilde{V}^{2}} \\
\varepsilon \equiv v \overline{\overline{\overline{\mathbf{J}}}(\tilde{\mathbf{V}}) \cdot \overline{\overrightarrow{\mathbf{J}}}(\tilde{\mathbf{V}})}
\end{gathered}
$$

where $\widetilde{\mathbf{V}}$ is the fluctuating velocity vector, $\overline{\overline{\mathbf{J}}}(\widetilde{\mathbf{V}})$ is its Jacobian tensor, and the overscore denotes an ensemble mean.

The eddy-viscosity model that is the foundation for all commonly used $k-\varepsilon, k-\omega$, and $k-\zeta$ turbulence models is

$$
v_{t}=C_{\mu} k^{2} / \varepsilon
$$

where $C_{\mu}$ is a dimensionless closure coefficient that is nearly universally accepted as being equal to 0.09 . The $k-\varepsilon$ turbulence models use Eq. (3) directly. The $k$ - $\omega$ turbulence models use the change of variables $\omega \equiv \varepsilon /\left(C_{\mu} k\right)$ to transform Eq. (3) to the equivalent relation given by $v_{t}=k / \omega$. Similarly, the $k-\zeta$ turbulence models use the change of variables $\zeta \equiv \varepsilon / v$ to transform Eq. (3) to its $k-\zeta$ equivalent, $v_{t}=C_{\mu} k^{2} /(v \zeta)$. The commonly used $k-\varepsilon, k-\omega$, and $k-\zeta$ turbulence models are all based on the hypothesis that the characteristic length scale for turbulent transport is proportional to the characteristic length scale for turbulent-energy dissipation.

The turbulent-energy-transport equation that is used in the traditional $k-\varepsilon, k-\omega$, and $k-\zeta$ turbulence models is commonly developed from the Navier-Stokes equations and the definition of the specific Reynolds stress tensor (i.e., the Reynolds stress tensor divided by the fluid density),

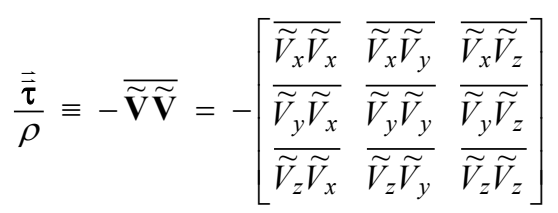

Multiplying the vector Navier-Stokes equation by the fluctuating velocity vector, taking the ensemble average, and adding the resulting tensor equation to its transpose yields a differential transport equation for the Reynolds stress tensor. From the definitions in Eqs. (1) and (4), the turbulent kinetic energy per unit mass, $k$, is the negative of onehalf the trace of the specific Reynolds stress tensor. Hence, the turbulent-energy-transport equation is commonly obtained from the negative of one-half the trace of the specific Reynolds-stress-transport equation. For the case of compressible flow with constant dynamic viscosity, this yields

$$
\begin{array}{r}
\rho\left(\frac{\partial k}{\partial t}+\overline{\mathbf{V}} \cdot \nabla k\right)=\overline{\overline{\boldsymbol{\tau}}} \cdot \overline{\overrightarrow{\mathbf{J}}}(\overline{\mathbf{V}})-\mu \overline{\overline{\overrightarrow{\mathbf{J}}}(\tilde{\mathbf{V}}) \cdot \overline{\overrightarrow{\mathbf{J}}}(\tilde{\mathbf{V}})}+\overline{\widetilde{p}(\nabla \cdot \tilde{\mathbf{V}})}-\frac{1}{3} \mu \overline{(\nabla \cdot \tilde{\mathbf{V}})^{2}} \\
+\mu \nabla^{2} k-\nabla \cdot\left[\frac{1}{2} \rho \overline{\widetilde{V}^{2} \widetilde{\mathbf{V}}}+\overline{\widetilde{p} \tilde{\mathbf{V}}}-\frac{1}{3} \mu \overline{(\nabla \cdot \tilde{\mathbf{V}}) \tilde{\mathbf{V}}}\right]
\end{array}
$$


The $k-\varepsilon, k-\omega$, and $k-\zeta$ turbulence models that utilize a version of the turbulent-kinetic-energy-transport equation given by Eq. (5) are based on the approximation that the turbulence parameter $\varepsilon$, which is defined in Eq. (2), is the dissipation of turbulent kinetic energy per unit mass. In general, this is not the case.

The turbulent-energy-transport equation can be alternately developed from the mechanical energy equation, which is obtained by taking the dot product of the fluid velocity vector with the Navier-Stokes equations written in vector form. Hence, the turbulent-energy-transport equation can be written $a^{2}$

$$
\begin{aligned}
& \rho\left(\frac{\partial k}{\partial t}+\overline{\mathbf{V}} \cdot \nabla k\right)=\overline{\bar{\tau}} \cdot \mathbf{J}(\overline{\mathbf{V}})-2 \mu\left[\overline{\overline{\overrightarrow{\mathbf{S}}}(\tilde{\mathbf{V}}) \cdot \overrightarrow{\overrightarrow{\mathbf{S}}}(\tilde{\mathbf{V}})}-\frac{1}{3} \overline{(\nabla \cdot \tilde{\mathbf{V}})^{2}}\right]+\overline{\tilde{p}(\nabla \cdot \tilde{\mathbf{V}})} \\
& +\nabla \cdot(\mu \nabla k-v \nabla \cdot \overline{\bar{\tau}})-\nabla \cdot\left[\frac{1}{2} \rho \overline{\widetilde{V}^{2} \widetilde{\mathbf{V}}}+\overline{\widetilde{p} \widetilde{\mathbf{V}}}+\frac{2}{3} \mu \overline{(\nabla \cdot \tilde{\mathbf{V}}) \tilde{\mathbf{V}}}\right]
\end{aligned}
$$

The first three terms on the right-hand side of Eq. (6) are exactly the volumetric production, viscous dissipation, and pressure dilatation for turbulent kinetic energy, respectively. The next term is the molecular transport of turbulent kinetic energy per unit volume. The last term on the right-hand side of Eq. (6) accounts for the volumetric turbulent transport of turbulent kinetic energy. The only approximation that was made in the development of Eq. (6) is that of a Newtonian fluid.

It is important to recognize from Eq. (6) that molecular transport of turbulent kinetic energy is not a simple gradient diffusion process. The contribution from the first part of the molecular transport term, $\mu \nabla k$, is gradient diffusion. However, the contribution from the remaining portion of this term, $v \nabla \cdot \overrightarrow{\bar{\tau}}$, is not necessarily gradient diffusion. Accordingly, even if we accept the Boussinesq analogy between molecular and turbulent transport, we should not expect turbulent transport of kinetic energy to be a simple gradient diffusion process in general.

Applying Boussinesq's analogy between molecular and turbulent transport to Eq. (6) results in an alternate version of the Boussinesq-based turbulent-energy-transport equation, ${ }^{2}$

$$
\begin{aligned}
\rho\left(\frac{\partial k}{\partial t}+\overline{\mathbf{V}} \cdot \nabla k\right) & =2 \mu_{t} \overline{\overrightarrow{\mathbf{S}}}(\overline{\mathbf{V}}) \cdot \overline{\overrightarrow{\mathbf{S}}}(\overline{\mathbf{V}})-\frac{2}{3}\left(\rho_{k}+\mu_{t} \nabla \cdot \overline{\mathbf{V}}\right) \nabla \cdot \overline{\mathbf{V}}-2 \mu\left[\overline{\overline{\mathbf{S}}}(\tilde{\mathbf{V}}) \cdot \overline{\overrightarrow{\mathbf{S}}}(\tilde{\mathbf{V}})-\frac{1}{3} \overline{(\nabla \cdot \tilde{\mathbf{V}})^{2}}\right] \\
& +\overline{\widetilde{p}(\nabla \cdot \tilde{\mathbf{V}})}+\nabla \cdot\left(\left(v+v_{t} / \sigma_{k}\right)\left\{\rho \nabla k+\frac{2}{3} \nabla\left(\rho k+\mu_{t} \nabla \cdot \overline{\mathbf{V}}\right)-2 \nabla \cdot\left[\mu_{t} \overline{\overrightarrow{\mathbf{S}}}(\overline{\mathbf{V}})\right]\right\}\right)
\end{aligned}
$$

For incompressible flow, Eq. (7) reduces to

$$
\frac{\partial k}{\partial t}+\overline{\mathbf{V}} \cdot \nabla k=2 v_{t} \overline{\overline{\mathbf{S}}}(\overline{\mathbf{V}}) \cdot \overline{\overrightarrow{\mathbf{S}}}(\overline{\mathbf{V}})-2 v \overline{\overline{\overrightarrow{\mathbf{S}}}(\tilde{\mathbf{V}}) \cdot \overline{\overrightarrow{\mathbf{S}}}(\tilde{\mathbf{V}})}+\nabla \cdot\left(\left(v+v_{t} / \sigma_{k}\right)\left\{\frac{5}{3} \nabla k-2 \nabla \cdot\left[v_{t} \overline{\overline{\mathbf{S}}}(\overline{\mathbf{V}})\right]\right\}\right)
$$

Although the third term on the right-hand side of Eq. (7) is exactly the volumetric dissipation of turbulent kinetic energy, it remains an unknown function of the turbulent velocity fluctuations. To close this formulation, we must have an additional equation to relate this dissipation to the other turbulence parameters and the mean flow.

As an alternative to traditional $k-\varepsilon$ turbulence models, the dissipation term that appears in Eqs. (6) and (7) can be mathematically rearranged. The exact turbulent-energy dissipation per unit mass can be written as ${ }^{2}$

$$
\begin{aligned}
\widetilde{\mathcal{\varepsilon}} & \equiv 2 v\left[\overline{\overline{\mathbf{S}}(\tilde{\mathbf{V}}) \cdot \overline{\overline{\mathbf{S}}}(\tilde{\mathbf{V}})}-\frac{1}{3} \overline{(\nabla \cdot \tilde{\mathbf{V}})^{2}}\right] \\
& =v\left\{\widetilde{\omega}^{2}+\frac{4}{3} \overline{(\nabla \cdot \tilde{\mathbf{V}})^{2}}-2 \nabla \cdot[(\nabla \cdot \overline{\bar{\tau}}) / \rho+\overline{(\nabla \cdot \tilde{\mathbf{V}}) \tilde{\mathbf{V}}}]\right\}
\end{aligned}
$$

where $\widetilde{\omega}$ is the RMS fluctuating vorticity,

$$
\widetilde{\omega}^{2} \equiv \overline{(\nabla \times \widetilde{\mathbf{V}}) \cdot(\nabla \times \widetilde{\mathbf{V}})}
$$

Hence, the turbulent-energy-transport equation can be written as

$$
\begin{array}{r}
\rho\left(\frac{\partial k}{\partial t}+\overline{\mathbf{V}} \cdot \nabla k\right)=\overline{\bar{\tau}} \cdot \mathbf{J}(\overline{\mathbf{V}})-\mu\left\{\widetilde{\omega}^{2}+\frac{4}{3} \overline{(\nabla \cdot \tilde{\mathbf{V}})^{2}}-2 \nabla \cdot[(\nabla \cdot \overline{\bar{\tau}}) / \rho+\overline{(\nabla \cdot \tilde{\mathbf{V}}) \tilde{\mathbf{V}}}]\right\} \\
+\overline{\widetilde{p}(\nabla \cdot \tilde{\mathbf{V}})}+\nabla \cdot\left[(\mu \nabla k-v \nabla \cdot \overline{\bar{\tau}})-\frac{1}{2} \rho \overline{\widetilde{V}^{2} \widetilde{\mathbf{V}}}-\overline{\widetilde{p} \widetilde{\mathbf{V}}}-\frac{2}{3} \mu \overline{(\nabla \cdot \tilde{\mathbf{V}}) \tilde{\mathbf{V}}}\right]
\end{array}
$$


Because only mathematical identities were used to obtain Eq. (11) from Eq. (6), these two transport equations are mathematically equivalent. The only approximation that was made in the development of Eq. (11) is that of a Newtonian fluid.

Applying the Boussinesq analogy between molecular and turbulent transport of turbulent kinetic energy to Eq. (11), Phillips ${ }^{2}$ developed the Boussinesq-transport equation

$$
\begin{aligned}
\rho\left(\frac{\partial k}{\partial t}+\overline{\mathbf{V}} \cdot \nabla k\right)= & 2 \mu_{t} \overrightarrow{\overrightarrow{\mathbf{S}}}(\overline{\mathbf{V}}) \cdot \overrightarrow{\overline{\mathbf{S}}}(\overline{\mathbf{V}})-\frac{2}{3}\left(\rho k+\mu_{t} \nabla \cdot \overline{\mathbf{V}}\right) \nabla \cdot \overline{\mathbf{V}} \\
& -\mu \widetilde{\omega}^{2}-4 \mu \nabla \cdot\left(\left\{\frac{1}{3} \nabla\left(\rho k+\mu_{t} \nabla \cdot \overline{\mathbf{V}}\right)-\nabla \cdot\left[\mu_{t} \overline{\overline{\mathbf{S}}}(\overline{\mathbf{V}})\right]\right\} / \rho\right) \\
& +\nabla \cdot\left(\left(v+v_{t} / \sigma_{k}\right)\left\{\rho \nabla k+\frac{2}{3} \nabla\left(\rho k+\mu_{t} \nabla \cdot \overline{\mathbf{V}}\right)-2 \nabla \cdot\left[\mu_{t} \overline{\overrightarrow{\mathbf{S}}}(\overline{\mathbf{V}})\right]\right\}\right)
\end{aligned}
$$

The three lines on the right-hand side of Eq. (12) are production, dissipation, and the combination of molecular and turbulent transport, respectively. Completing this formulation requires two additional equations relating the eddy viscosity, $v_{t}$, the specific turbulent kinetic energy, $k$, and the RMS fluctuating vorticity, $\widetilde{\omega}$.

Following an analogy with the kinetic theory of gases, Phillips ${ }^{2}$ proposed an alternative to traditional $k-\varepsilon, k-\omega$, and $k-\zeta$ turbulence models by using the RMS fluctuating vorticity, $\widetilde{\omega}$, as the characteristic frequency associated with turbulent transport. This produces the foundation for an energy-vorticity turbulence model, which is based on the following two equations for incompressible flow; an algebraic equation for the kinematic eddy viscosity,

$$
v_{t}=C_{v} k / \widetilde{\omega}
$$

and the turbulent-energy-transport equation from Eq. (12),

$$
\begin{array}{r}
\frac{\partial k}{\partial t}+\overline{\mathbf{V}} \cdot \nabla k=2 v_{t} \overrightarrow{\overrightarrow{\mathbf{S}}}(\overline{\mathbf{V}}) \cdot \overrightarrow{\overrightarrow{\mathbf{S}}}(\overline{\mathbf{V}})-v\left(\widetilde{\omega}^{2}+4 \nabla \cdot\left\{\frac{1}{3} \nabla k-\nabla \cdot\left[v_{t} \overrightarrow{\overrightarrow{\mathbf{S}}}(\overline{\mathbf{V}})\right]\right\}\right) \\
+\nabla \cdot\left(\left(v+v_{t} / \sigma_{k}\right)\left\{\frac{5}{3} \nabla k-2 \nabla \cdot\left[v_{t} \overrightarrow{\overrightarrow{\mathbf{S}}}(\overline{\mathbf{V}})\right]\right\}\right)
\end{array}
$$

where $C_{V}$ and $\sigma_{k}$ are dimensionless closure coefficients. Completing this formulation requires one additional equation relating the eddy viscosity, $v_{t}$, the specific turbulent kinetic energy, $k$, the RMS fluctuating vorticity, $\widetilde{\omega}$, and the mean velocity vector, $\overline{\mathbf{V}}$. The formulation could be completed by including a vorticity-transport equation for the RMS fluctuating vorticity, $\widetilde{\omega}$.

Equations (13) and (14) are easily recast in terms of the mean fluctuating enstrophy, which is commonly denoted as $\zeta$ and defined to be the mean squared magnitude of the fluctuating vorticity,

$$
\zeta \equiv \widetilde{\omega}^{2} \equiv \overline{(\nabla \times \widetilde{\mathbf{V}}) \cdot(\nabla \times \widetilde{\mathbf{V}})}
$$

Using Eq. (15) in Eqs. (13) and (14) produces the foundation for an energy-enstrophy turbulence model, which is based on the following two equations for incompressible flow; ${ }^{2}$ an algebraic equation for the kinematic eddy viscosity,

$$
v_{t}=C_{v} k / \zeta^{1 / 2}
$$

and the turbulent-energy-transport equation obtained by applying Eq. (15) to Eq. (14),

$$
\begin{array}{r}
\frac{\partial k}{\partial t}+\overline{\mathbf{V}} \cdot \nabla k=2 v_{t} \overline{\overrightarrow{\mathbf{S}}}(\overline{\mathbf{V}}) \cdot \overrightarrow{\overrightarrow{\mathbf{S}}}(\overline{\mathbf{V}})-v\left(\zeta+4 \nabla \cdot\left\{\frac{1}{3} \nabla k-\nabla \cdot\left[v_{t} \overrightarrow{\overrightarrow{\mathbf{S}}}(\overline{\mathbf{V}})\right]\right\}\right) \\
+\nabla \cdot\left(\left(v+v_{t} / \sigma_{k}\right)\left\{\frac{5}{3} \nabla k-2 \nabla \cdot\left[v_{t} \overline{\overrightarrow{\mathbf{S}}}(\overline{\mathbf{V}})\right]\right\}\right)
\end{array}
$$

Completing this formulation requires one additional equation relating the eddy viscosity, $v_{t}$, the specific turbulent kinetic energy, $k$, the mean fluctuating enstrophy, $\zeta$, and the mean velocity vector, $\overline{\mathbf{V}}$. This formulation could be completed by including the enstrophy-transport equation from the traditional Robinson-Hassan $k-\zeta$ turbulence model. ${ }^{3,4}$ Perhaps a more promising enstrophy-transport equation for closing the proposed $k-\zeta$ turbulence model can be obtained from the DNS-based solenoidal-dissipation model of Kreuzinger, Friedrich, and Gatski. ${ }^{5}$ 
Equations (13) and (14) can also be reparameterized using a turbulent-transport length scale in place of the RMS fluctuating vorticity ${ }^{2}$

$$
\lambda \equiv C_{V} \frac{k^{1 / 2}}{\widetilde{\omega}}=C_{V} \sqrt{\frac{\overline{\tilde{\mathbf{V}} \cdot \widetilde{\mathbf{V}}}}{2 \overline{(\nabla \times \tilde{\mathbf{V}}) \cdot(\nabla \times \tilde{\mathbf{V}})}}}
$$

where the vorticity-based turbulent-transport length scale, $\lambda$, will be referred to here as the mean vortex wavelength. Applying this change of variables to Eqs. (13) and (14) produces the foundation for a $\boldsymbol{k}$ - $\boldsymbol{\lambda}$ turbulence model, which is based on the following two equations for incompressible flow; an algebraic equation for the kinematic eddy viscosity,

$$
v_{t}=\lambda k^{1 / 2}
$$

and the turbulent-energy-transport equation obtained by applying Eq. (18) to Eq. (14),

$$
\begin{aligned}
\frac{\partial k}{\partial t}+\overline{\mathbf{V}} \cdot \nabla k=2 v_{t} \overline{\overrightarrow{\mathbf{S}}}(\overline{\mathbf{V}}) \cdot \overline{\overrightarrow{\mathbf{S}}}(\overline{\mathbf{V}}) & -v\left(C_{\lambda} k / \lambda^{2}+4 \nabla \cdot\left\{\frac{1}{3} \nabla k-\nabla \cdot\left[v_{t} \overline{\overline{\mathbf{S}}}(\overline{\mathbf{V}})\right]\right\}\right) \\
+ & \nabla \cdot\left(\left(v+v_{t} / \sigma_{k}\right)\left\{\frac{5}{3} \nabla k-2 \nabla \cdot\left[v_{t} \overline{\overrightarrow{\mathbf{S}}}(\overline{\mathbf{V}})\right]\right\}\right)
\end{aligned}
$$

where $C_{\lambda}$ and $\sigma_{k}$ are dimensionless closure coefficients, $C_{\lambda} \equiv C_{v}^{2}$. Completing this formulation requires one additional equation relating the eddy viscosity, $v_{t}$, the specific turbulent kinetic energy, $k$, the mean vortex wavelength, $\lambda$, and the mean velocity vector, $\overline{\mathbf{V}}$.

To complete a two-equation energy-vorticity turbulence model, a second transport equation like that suggested by Kreuzinger, Friedrich, and Gatski ${ }^{5}$ is required and the closure coefficients in the turbulent-energy-transport equation must be evaluated. Closure coefficients are typically evaluated from well established experimental data. One case that is very well documented is fully developed flow in a pipe. Because the friction factor becomes independent of the Reynolds number for fully rough flow in a given pipe, estimating the closure coefficients from data for rough surfaces might be more straightforward than using data for smooth surfaces. In the following sections, the mean-vortex-wavelength and RMS-fluctuating-vorticity profiles for fully developed, fully rough pipe flow are inferred from Eqs. (19) and (20) combined with experimental data and well established empirical correlations. This will provide some insight into the nature of the energy-vorticity turbulence variables and establish certain relations between the closure coefficients.

\section{Friction Factor in Fully Rough Pipe Flow}

The foundation for what is known today about turbulent flow in rough pipes is the semi-empirical mixing-length theory developed by Ludwig Prandtl and his students. The rough-wall version of Prandtl's mixing-length theory is based on experimental data collected by Prandtl's famous student Johann Nikuradse ${ }^{6}$ using pipes roughened artificially with uniform grains of sand. Nikuradse's data for fully rough pipe flow is also the foundation for the empirical relations commonly used today for predicting pressure losses in rough pipes; including the Colebrook equation, ${ }^{7}$ which was used to generate the well known Moody chart. ${ }^{8}$ Based on his experimental results for the Darcy friction factor, which is four times the Fanning friction factor often called the skin-friction coefficient, Nikuradse ${ }^{6}$ proposed using the following empirical correlation for fully rough pipe flow:

$$
\text { Darcy friction factor } \equiv 4 C_{f} \equiv 4 \frac{\tau_{w}}{\frac{1}{2} \rho V_{m}^{2}}=\left[2.00 \log _{10}\left(R / k_{s}\right)+1.74\right]^{-2}
$$

where $R$ is the pipe radius, $V_{m}$ is the bulk velocity, and $k_{s}$ is the roughness element size defined to be the screen mesh size of the sieve that Nikuradse used to sift the sand. Equation (21) provided a key result in the development of our current capability to predict pressure losses for turbulent flow through rough pipes, and it is referred to herein as the Nikuradse equation. Equation (21) was the starting point for the development the Colebrook equation ${ }^{7}$ and the associated Moody chart. ${ }^{8}$ Thus, the Colebrook equation and the Moody chart assume the validity of the Nikuradse equation. 
The Nikuradse equation is often presented in a form that differs slightly from Eq. (21). In the original work by Nikuradse ${ }^{6}$ and its subsequent presentation by another of Prandtl's famous students, Hermann Schlichting, ${ }^{9}$ the pipe roughness was characterized using the dimensionless roughness ratio, $R / k_{s}$. When Colebrook ${ }^{7}$ applied the Nikuradse equation to his work, he chose to characterize pipe roughness using the dimensionless relative roughness, $k_{s} / D$, where $D$ is the pipe diameter. Thus, the Nikuradse equation was rearranged by Colebrook into the form

$$
4 C_{f}=\left[2.00 \log _{10}\left(\frac{10^{1.74 / 2.00} / 2}{k_{s} / 2 R}\right)\right]^{-2}
$$

Rounding the constants to two significant digits yields the most widely accepted form of the Nikuradse equation,

$$
4 C_{f}=\left[2.0 \log _{10}\left(\frac{3.7}{k_{s} / D}\right)\right]^{-2}
$$

The validity of Eq. (23) is so widely accepted that today it has become the definition of surface roughness. The roughness of any surface is typically defined in terms of the equivalent sand-grain roughness first introduced by Schlichting, ${ }^{10}$ which is defined to be the value of $k_{s}$ that gives the correct fully rough limit for the friction factor when inserted into Eq. (23). It should be noted that when Moody ${ }^{8}$ used the relation developed by Colebrook ${ }^{7}$ to generate the well known Moody chart, he used the symbol $\varepsilon$ to denote Nikuradse's sand-grain roughness. Here we will continue to use $k_{s}$ to signify the equivalent sand-grain roughness, following what was done by Nikuradse, ${ }^{6}$ Schlichting, ${ }^{10}$ and Colebrook. ${ }^{7}$

The Nikuradse equation expressed equivalently in Eqs. (21) and (23) provides an accurate means for predicting the Darcy friction factor when the Reynolds number is large enough so that the friction factor becomes independent of the molecular viscosity. However, the Nikuradse equation alone provides no information regarding how large the Reynolds number must be to make this empirical correlation valid. From Nikuradse's data ${ }^{6}$ on artificially roughened pipes, it is commonly accepted that this correlation for fully rough flow is valid whenever the Reynolds number based on the shear velocity $u_{\tau}$ and the equivalent sand-grain roughness $k_{s}$, usually called the roughness Reynolds number, is greater than about 70 . The Nikuradse equation can be used as a reference when calculating the Darcy friction factor for fully rough pipe flows.

Of the conventional $k-\varepsilon, k-\omega$, and $k-\zeta$ turbulence models, only $k$ - $\omega$ models are capable of implementing roughwall boundary conditions without employing wall functions. However, even the current $k$ - $\omega$ models are not capable of predicting friction factors that agree with experimental data at very high roughness Reynolds numbers, where the molecular viscosity is negligible compared to the eddy viscosity throughout the pipe. The effects of surface roughness are incorporated into conventional $k$ - $\omega$ turbulence models by altering the surface boundary condition on $\omega$. For example, with his 1998 model, Wilcox ${ }^{11}$ suggests using the rough-wall boundary condition

$$
\left.\omega^{+}\right|_{y^{+}=0}= \begin{cases}\left(50 / k_{s}^{+}\right)^{2}, & k_{s}^{+} \leq 25 \\ 100 / k_{s}^{+}, & k_{s}^{+} \geq 25\end{cases}
$$

where $k_{s}^{+}$is the roughness Reynolds number. For his 2006 model, Wilcox ${ }^{12}$ recommends using

$$
\left.\omega^{+}\right|_{y^{+}=0}= \begin{cases}\left(200 / k_{s}^{+}\right)^{2}, & k_{s}^{+} \leq 5 \\ 100 / k_{s}^{+}+\left[\left(200 / k_{s}^{+}\right)^{2}-100 / k_{s}^{+}\right] \exp \left(5-k_{s}^{+}\right), & k_{s}^{+} \geq 5\end{cases}
$$

A comparison between experimental data ${ }^{6,13}$ and results obtained from the Wilcox $1998 k$ - $\omega$ model $^{11}$ is shown in Fig. 1 for roughness Reynolds numbers greater than 70. The Darcy friction factors obtained from the Wilcox 2006 $k$ - $\omega$ model $^{12}$ are shown in Fig. 2. To assure that the results shown in Figs. 1 and 2 are grid resolved, converged solutions were obtained on coarse, medium, and fine grids, containing 401, 801, and 1601 nodes, respectively. The values of $y^{+}$at the first node from the pipe wall in these coarse, medium, and fine grids were 0.4, 0.2, and 0.1, respectively. The Richardson ${ }^{14}$ extrapolation was then used with these three solutions to obtain the results shown in Figs. 1 and 2. The maximum difference observed between the Richardson ${ }^{14}$ extrapolation and the solution obtained on the fine grid was 1.3 percent for the 1998 model and 0.04 percent for the 2006 model. 
The 1998 and 2006 software provided by Wilcox ${ }^{11,12}$ would not converge for high roughness Reynolds numbers, which encompasses the region to the right of the right-hand dashed curves in Figs. 1 and 2. Along these curves the ratio of the eddy viscosity near the pipe wall to the molecular viscosity is nearly constant at about 1.7 for the 1998 model and 1.3 for the 2006 model. As a result, the Wilcox 1998 and $2006 k$ - $\omega$ models cannot be used to predict the distributions of the turbulence variables in the fully rough region, where the molecular viscosity is negligible compared to the eddy viscosity throughout the pipe. One important objective for an improved rough-wall turbulence model should be the capability to accurately predict the Darcy friction factor at high roughness Reynolds numbers.

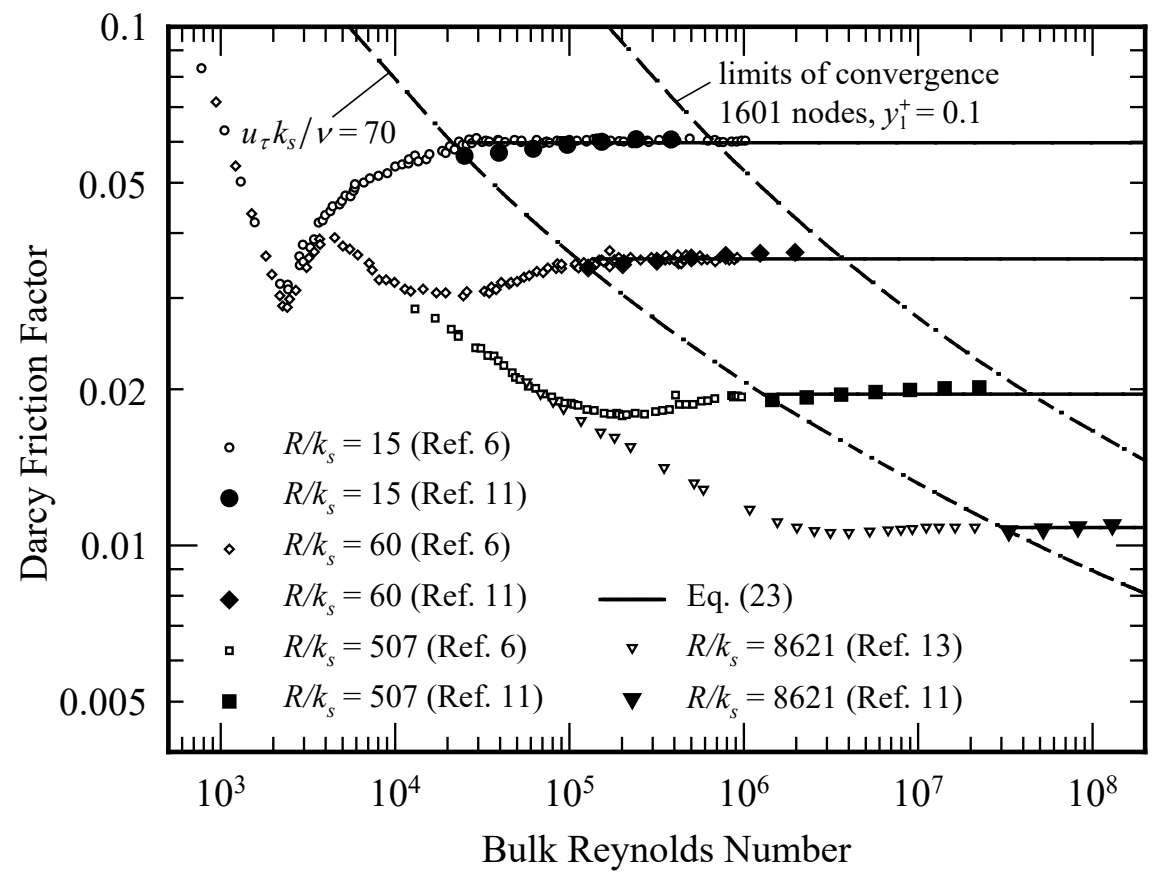

Figure 1. Darcy friction factor, as predicted from the Wilcox $1998 \boldsymbol{k}$ - $\omega$ model. ${ }^{11}$

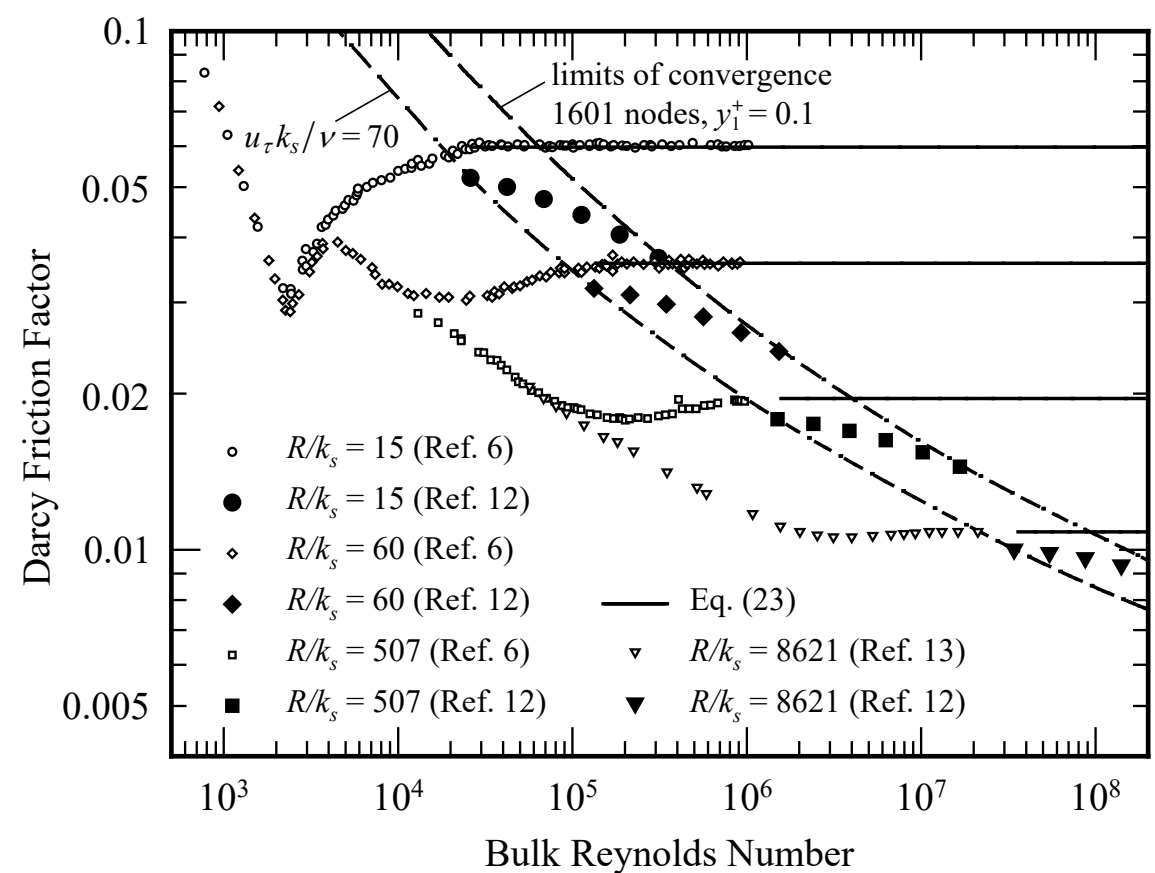

Figure 2. Darcy friction factor, as predicted from the Wilcox $2006 \boldsymbol{k}$ - $\omega$ model. $^{12}$ 


\section{Mean-Velocity Profiles in Fully Rough Pipe Flow}

A sensitive indicator for the effect of surface roughness in pipe flow is given by the behavior of the mean velocity profile. Mean-velocity-profile measurements taken by Nikuradse ${ }^{6}$ in the fully rough limit were found to be in excellent agreement with the empirical correlation

$$
\frac{\bar{V}_{z}}{u_{\tau}}=2.5 \ln \left(\frac{y}{k_{s}}\right)+8.5
$$

where $y$ is the normal coordinate measured into the fluid from the pipe wall. Equation (26) is commonly referred to as the law of the wall for fully rough pipe flow. A comparison between Eq. (26) and experimental data collected by Nikuradse ${ }^{6}$ is shown in Fig. 3.

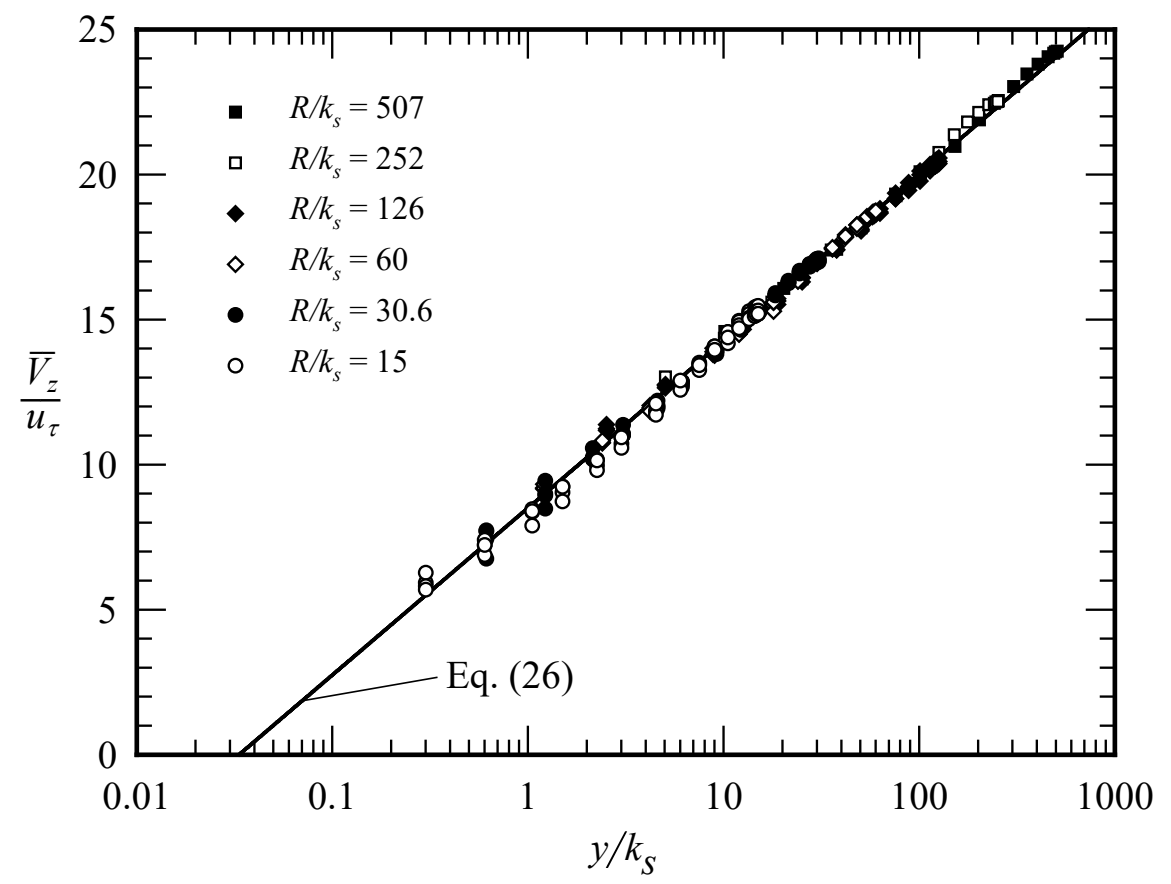

Figure 3. Experimental velocity profiles in rough pipes at high Reynolds numbers. ${ }^{6}$

Obviously, Eq. (26) does not apply over the entire flow field from the pipe wall to the centerline, because it satisfies neither the no-slip boundary condition at the pipe wall nor the symmetry boundary condition at the pipe centerline. In order to satisfy these two boundary conditions and provide better agreement with Nikuradse's experimental data while leaving the integral of the velocity profile unchanged, a correction is applied to the law of the wall that is given by Eq. (26), ${ }^{15}$

$$
\frac{\bar{V}_{z}}{V_{m}}=\frac{\hat{R}_{s}^{2} \ln \left(\hat{R}_{s} \hat{y}+1\right)}{D_{0}}+\delta
$$

or

$$
\frac{\bar{V}_{z}}{u_{\tau}}=\frac{1}{\kappa} \ln \left(\hat{R}_{s} \hat{y}+1\right)+\frac{D_{0} \delta}{\kappa \hat{R}_{s}^{2}}
$$

A seventh-order corrective function $\delta$ is given by ${ }^{15}$

$$
\delta=C_{0}+C_{1} \hat{r}+C_{2} \hat{r}^{2}+C_{3} \hat{r}^{3}+C_{4} \hat{r}^{4}+C_{5} \hat{r}^{5}+C_{6} \hat{r}^{6}+C_{7} \hat{r}^{7}
$$


where

$$
\begin{aligned}
& D_{0}=\left(\hat{R}_{s}+1\right)^{2}\left[\ln \left(\hat{R}_{s}+1\right)-\frac{3}{2}\right]+2 \hat{R}_{s}+\frac{3}{2}, \quad C_{0}=1.2\left(\frac{k_{s}}{R}\right)^{1.4} \\
& C_{1}=\frac{\hat{R}_{s}^{3}}{\left(\hat{R}_{s}+1\right) D_{0}}, \quad C_{2}=\frac{\hat{R}_{s}^{2}}{2 D_{0}}\left[\frac{\hat{R}_{s}^{2}}{\left(\hat{R}_{s}+1\right)^{2}}-\frac{\kappa}{0.056}\right], \quad C_{3}=\frac{\hat{R}_{s}^{5}}{3\left(\hat{R}_{s}+1\right)^{3} D_{0}} \\
& D_{1}=-C_{0}-C_{1}-C_{2}-C_{3}-C_{7}, \quad D_{2}=-C_{1}-2 C_{2}-3 C_{3}-7 C_{7} \\
& D_{3}=-C_{0} / 2-C_{1} / 3-C_{2} / 4-C_{3} / 5-C_{7} / 9 \\
& C_{4}=-39 D_{1}+3 D_{2}+168 D_{3}, \quad C_{5}=84 D_{1}-7 D_{2}-336 D_{3} \\
& C_{6}=-44 D_{1}+4 D_{2}+168 D_{3}, \quad C_{7}=-0.65 \\
& \hat{R}_{s}=R /\left(\gamma k_{s}\right), \quad \hat{y}=y / R, \quad \hat{r}=1-\hat{y}, \quad \kappa=0.403, \quad \gamma=0.0324
\end{aligned}
$$

The eight coefficients in Eq. (29) that are given in Eq. (30) were adjusted to optimize the fit with Nikuradse's velocity profile data for fully rough flow while satisfying the physical constraints imposed on the empirical function $\delta$ by the symmetry and no-slip boundary conditions. The values used for the von Kármán constant, $\kappa$, and the Nikuradse constant, $\gamma$, were also adjusted to optimize the fit with Nikuradse's velocity profile data. Hence, we are using slightly different values for these constants than the values $\kappa=0.40$ and $\gamma=0.033$, which were used by Nikuradse ${ }^{6}$ and Schlichting. ${ }^{9}$ The resulting velocity profiles were found to be in good agreement with Nikuradse's experimental data on fully rough pipe flow. As an example, Fig. 4 shows the velocity obtained from Eqs. (27)-(30) compared to the law of the wall and Nikuradse's experimental data for a roughness ratio, $R / k_{s}$, of 15 . Because the velocity profiles obtained from Eqs. (27)-(30) satisfy the physical boundary conditions and show better agreement with experimental data, they can be used in place of Eq. (26) as an improved reference for the mean velocity profiles when evaluating turbulence model closure coefficients for fully rough pipe flows.

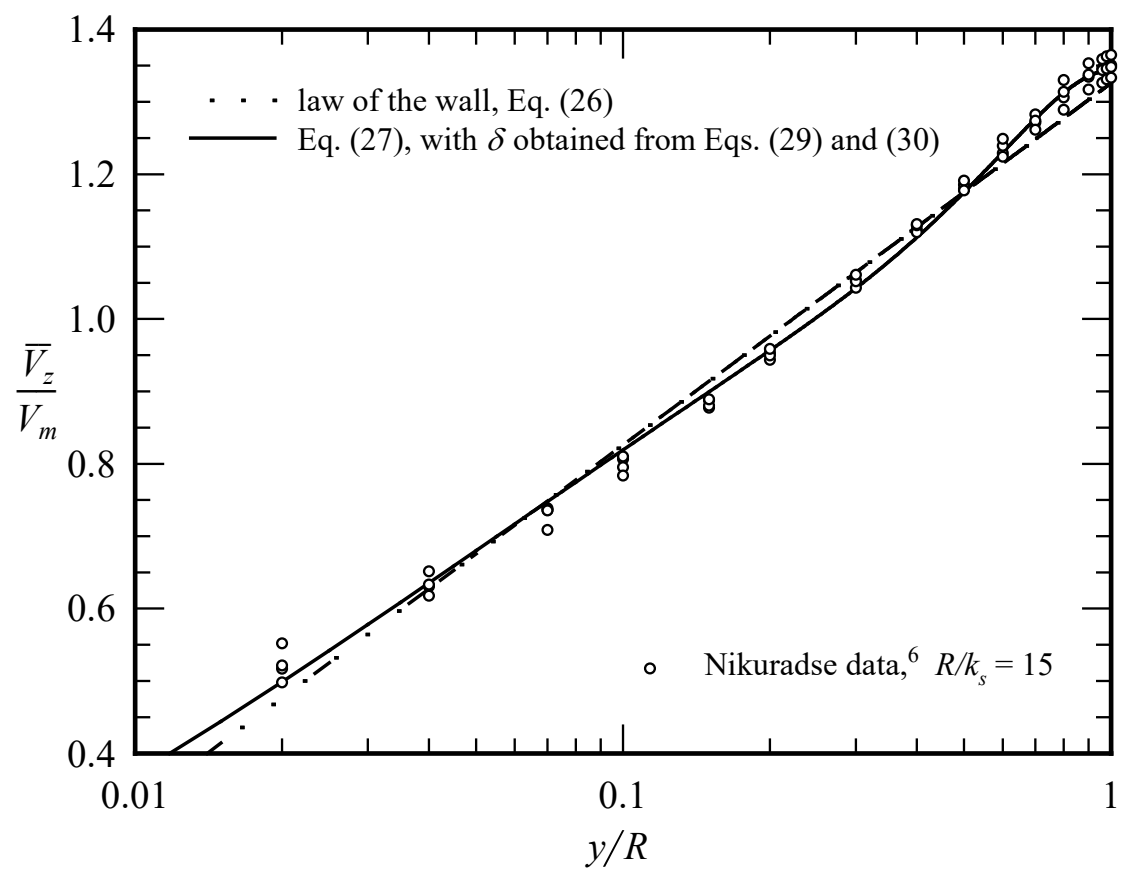

Figure 4. Velocity profiles compared to the law of the wall and Nikuradse's experimental data. ${ }^{6}$

\section{Mean-Vortex-Wavelength Profiles in Fully Rough Pipe Flow}

In order to complete the formulation given by Eqs. (19) and (20), an equation is needed for the mean vortex wavelength, $\lambda$. To provide some insight into the nature of the mean vortex wavelength and the RMS fluctuating 
vorticity, Eqs. (19) and (20) can be used in combination with empirical correlations for the friction-factor and the mean-velocity profiles to infer the mean-vortex-wavelength and RMS-fluctuating-vorticity distributions for fully developed, fully rough pipe flow.

From Eq. (20), the turbulent-kinetic-energy transport equation for axisymmetric fully developed pipe flow is given by

$$
\frac{1}{r} \frac{d}{d r}\left[-\left(\frac{v}{3}+\frac{5 v_{t}}{3 \sigma_{k}}\right) r \frac{d k}{d r}\right]=v_{t}\left(\frac{u_{\tau}^{2} r}{\left(v+v_{t}\right) R}\right)^{2}-C_{\lambda} \frac{v k}{\lambda^{2}}
$$

where $v_{t}=\lambda k^{1 / 2}$. This equation contains two unknown closure coefficients $\sigma_{k}$ and $C_{\lambda}$. These two coefficients must be dimensionless universal constants.

Equation (31) requires a wall boundary condition for the turbulent kinetic energy. The specific turbulent kinetic energy at a rough wall should be proportional to the shear velocity squared,

$$
\left.k\right|_{r=R}=k_{\mathrm{wall}}^{+} u_{\tau}^{2}
$$

where $k_{\mathrm{wal}}^{+}$is an unknown dimensionless proportionality coefficient. For incompressible flow, the dimensionless parameter, $k_{\mathrm{wal}}^{+}$, should be a unique function of the roughness Reynolds number. As the roughness Reynolds number approaches zero, $k_{\mathrm{w} \text { al }}^{+}$should also approach zero. When the roughness Reynolds number becomes large enough, the solution must be independent of molecular viscosity, i.e., independent of $u_{\tau} k_{s} / v$ at constant $k_{s} / R$. Hence, for fully rough flow, the dimensionless parameter, $k_{\mathrm{w} \text { a }}^{+}$, must approach a universal constant.

An algebraic function for the mean vortex wavelength in fully rough pipe flow is given by ${ }^{15}$

$$
\lambda=\left(A_{\lambda 0} k_{s}+A_{\lambda 1} y\right)\left(1-\frac{1}{2} \frac{y}{R}\right)\left[B_{\lambda 0}+B_{\lambda 1}\left(\frac{r}{R}\right)^{2}+B_{\lambda 2}\left(\frac{r}{R}\right)^{4}+\left(1-B_{\lambda 0}-B_{\lambda 1}-B_{\lambda 2}\right)\left(\frac{r}{R}\right)^{6}\right]
$$

where $y=R-r$. This equation contains five unknown coefficients $A_{\lambda 0}, A_{\lambda 1}, B_{\lambda 0}, B_{\lambda 1}$, and $B_{\lambda 2}$. At the wall, the mean-vortex-wavelength equation reduces to

$$
\left.\lambda\right|_{r=R}=A_{\lambda 0} k_{s}
$$

For fully rough pipe flow, the value of the mean vortex wavelength at the wall should depend only on the surface roughness $k_{s}$. Therefore, the coefficient $A \lambda_{0}$ should be a constant. The remaining four coefficients in the meanvortex-wavelength profile, $A_{\lambda_{1}}, B_{\lambda_{0}}, B_{\lambda_{1}}$, and $B_{\lambda_{2}}$, need not be constants, but can be functions of the flow parameters such as the Reynolds number and roughness ratio. Eight coefficients are associated with Eqs. (31)-(33); the two closure coefficients $\sigma_{k}$ and $C_{\lambda}$ from the turbulent-kinetic-energy transport equation, the proportionality coefficient $k_{\mathrm{wal}}^{+}$from the wall boundary condition for $k$, and the five coefficients $A_{\lambda_{0}}, A_{\lambda_{1}}, B \lambda_{0}, B \lambda_{1}, B_{\lambda_{2}}$ from the empirical algebraic relation for the mean vortex wavelength.

The eight coefficients $\sigma_{k}, C_{\lambda}, k_{\mathrm{wal}}^{+}, A \lambda_{0}, A \lambda_{1}, B \lambda_{0}, B \lambda_{1}, B \lambda_{2}$ were evaluated using computer optimization software. ${ }^{16}$ This optimization software minimizes a fitness parameter that quantifies how close the solution is to a target solution. The target solution is a weighted function based on the friction factor obtained from Eq. (23) and the velocity profile given by Eqs. (27)-(30). This fitness parameter was minimized over the Reynolds-number range starting at a roughness Reynolds number of 1000 and continuing up to a bulk Reynolds number as large as $2 \times 10^{9}$. The optimization software used to find the coefficients implements the BFGS algorithm, named after the work of Broyden, ${ }^{17}$ Fletcher ${ }^{18}$ Goldfarb, ${ }^{19}$ and Shanno. ${ }^{20}$ The resulting algebraic relation for the mean vortex wavelength was found to give good agreement with experimental data, provided that $\sigma_{k}$ is in the range 2.0 to $6.0, k_{\mathrm{wal}}^{+}$is in the range 0.05 to 1.0 , and the following relations between the coefficients are maintained;

$$
\begin{gathered}
C_{\lambda}=\left(1.143 \times 10^{-4} \sigma_{k}^{4}-2.157 \times 10^{-3} \sigma_{k}^{3}+1.545 \times 10^{-2} \sigma_{k}^{2}-5.315 \times 10^{-2} \sigma_{k}+8.365 \times 10^{-2}\right)^{2} \\
A_{\lambda 0}=-5.481 \times 10^{-6} \sigma_{k}^{4}+1.083 \times 10^{-4} \sigma_{k}^{3}-5.882 \times 10^{-4} \sigma_{k}^{2}+6.427 \times 10^{-5} \sigma_{k}+7.056 \times 10^{-3}
\end{gathered}
$$




$$
\begin{aligned}
& A_{\lambda 10}=6.055 \times 10^{-6} \sigma_{k}^{4}-1.746 \times 10^{-4} \sigma_{k}^{3}+1.708 \times 10^{-3} \sigma_{k}^{2}-7.802 \times 10^{-3} \sigma_{k}+2.362 \times 10^{-2} \\
& A_{\lambda 11}=1.643 \times 10^{-4} \sigma_{k}^{4}-3.288 \times 10^{-3} \sigma_{k}^{3}+2.367 \times 10^{-2} \sigma_{k}^{2}-7.482 \times 10^{-2} \sigma_{k}+1.374 \times 10^{-1} \\
& C_{4}=-3.625 \times 10^{-4} k_{\text {wall }}^{+2}-2.377 \times 10^{-4} k_{\text {wall }}^{+}+3.181 \times 10^{-4} \\
& C_{3}=6.746 \times 10^{-3} k_{\mathrm{wall}}^{+2}+3.503 \times 10^{-3} k_{\mathrm{wall}}^{+}-7.604 \times 10^{-3} \\
& C_{2}=-4.306 \times 10^{-2} k_{\text {wall }}^{+2}-2.005 \times 10^{-2} k_{\text {wall }}^{+}+5.737 \times 10^{-2} \\
& C_{1}=1.110 \times 10^{-1} k_{\mathrm{wall}}^{+2}+5.732 \times 10^{-2} k_{\mathrm{wall}}^{+}-1.577 \times 10^{-1} \\
& C_{0}=-9.789 \times 10^{-2} k_{\text {wall }}^{+2}-7.677 \times 10^{-2} k_{\text {wall }}^{+}+1.640 \times 10^{-1} \\
& A_{\lambda 12}=C_{4} \sigma_{k}^{4}+C_{3} \sigma_{k}^{3}+C_{2} \sigma_{k}^{2}+C_{1} \sigma_{k}+C_{0} \\
& A_{\lambda 13}=2.178 \times 10^{-4} \sigma_{k}^{4}-4.613 \times 10^{-3} \sigma_{k}^{3}+3.200 \times 10^{-2} \sigma_{k}^{2}-7.606 \times 10^{-2} \sigma_{k}+2.679 \times 10^{-1} \\
& B_{\lambda 00}=1.539 \times 10^{-3} \sigma_{k}^{4}-3.768 \times 10^{-2} \sigma_{k}^{3}+3.054 \times 10^{-1} \sigma_{k}^{2}-9.981 \times 10^{-1} \sigma_{k}+1.123 \\
& B_{\lambda 01}=-1.761 \times 10^{-3} \sigma_{k}^{4}+3.347 \times 10^{-2} \sigma_{k}^{3}-2.156 \times 10^{-1} \sigma_{k}^{2}+4.948 \times 10^{-1} \sigma_{k}+4.408 \times 10^{-1} \\
& C_{4}=4.596 \times 10^{-3} k_{\text {wall }}^{+2}+2.083 \times 10^{-3} k_{\text {wall }}^{+}-1.328 \times 10^{-2} \\
& C_{3}=-9.312 \times 10^{-2} k_{\text {wall }}^{+2}-2.857 \times 10^{-2} k_{\text {wall }}^{+}+2.759 \times 10^{-1} \\
& C_{2}=6.040 \times 10^{-1} k_{\text {wall }}^{+2}+1.876 \times 10^{-1} k_{\text {wall }}^{+}-2.008 \\
& C_{1}=-1.495 k_{\text {wall }}^{+2}-7.378 \times 10^{-1} k_{\text {wall }}^{+}+6.034 \\
& C_{0}=1.214 k_{\text {wall }}^{+2}+1.270 k_{\text {wall }}^{+}-7.287 \\
& B_{\lambda 02}=C_{4} \sigma_{k}^{4}+C_{3} \sigma_{k}^{3}+C_{2} \sigma_{k}^{2}+C_{1} \sigma_{k}+C_{0} \\
& B_{\lambda 03}=6.345 \times 10^{-4} \sigma_{k}^{4}-1.716 \times 10^{-2} \sigma_{k}^{3}+1.490 \times 10^{-1} \sigma_{k}^{2}-5.319 \times 10^{-1} \sigma_{k}+7.978 \times 10^{-1} \\
& B_{\lambda 10}=-1.013 \times 10^{-3} \sigma_{k}^{4}+1.915 \times 10^{-2} \sigma_{k}^{3}-1.222 \times 10^{-1} \sigma_{k}^{2}+2.553 \times 10^{-1} \sigma_{k}+1.436 \\
& B_{\lambda 11}=-6.621 \times 10^{-3} \sigma_{k}-13.83 \\
& C_{4}=-2.020 \times 10^{-3} k_{\text {wall }}^{+2}+4.328 \times 10^{-3} k_{\text {wall }}^{+}-1.395 \times 10^{-3} \\
& C_{3}=2.657 \times 10^{-2} k_{\text {wall }}^{+2}-6.414 \times 10^{-2} k_{\text {wall }}^{+}+2.362 \times 10^{-2} \\
& C_{2}=-1.199 \times 10^{-1} k_{\text {wall }}^{+2}+3.337 \times 10^{-1} k_{\text {wall }}^{+}-1.386 \times 10^{-1} \\
& C_{1}=2.194 \times 10^{-1} k_{\mathrm{wall}}^{+2}-7.081 \times 10^{-1} k_{\mathrm{wall}}^{+}+3.032 \times 10^{-1} \\
& C_{0}=-1.407 \times 10^{-1} k_{\text {wall }}^{+2}+4.584 \times 10^{-1} k_{\text {wall }}^{+}-8.085 \times 10^{-1} \\
& B_{\lambda 20}=C_{4} \sigma_{k}^{4}+C_{3} \sigma_{k}^{3}+C_{2} \sigma_{k}^{2}+C_{1} \sigma_{k}+C_{0} \\
& B_{\lambda 21}=-2.682 \times 10^{-3} \sigma_{k}+40.04
\end{aligned}
$$

The twelve constants $A_{\lambda 10}, A_{\lambda_{11}}, A_{\lambda 12}, A_{\lambda 13}, B_{\lambda 00}, B_{\lambda 01}, B_{\lambda 02}, B_{\lambda 03}, B_{\lambda 10}, B_{\lambda_{11}}, B_{\lambda 20}$, and $B_{\lambda_{21}}$ are related to the four coefficients $A \lambda_{1}, B \lambda_{0}, B \lambda_{1}, B \lambda_{2}$ according to 


$$
\begin{gathered}
A_{\lambda 1}=A_{\lambda 11}+A_{\lambda 12} \hat{k}_{s}+\left(A_{\lambda 10}-A_{\lambda 11}-A_{\lambda 12} \hat{k}_{s}\right) \exp \left(-\hat{k}_{s}^{A_{\lambda 13}}\right) \\
B_{\lambda 0}=B_{\lambda 01}+B_{\lambda 02} \hat{k}_{s}+\left(B_{\lambda 00}-B_{\lambda 01}-B_{\lambda 02} \hat{k}_{s}\right) \exp \left(-\hat{k}_{s}^{B_{\lambda 03}}\right) \\
B_{\lambda 1}=B_{\lambda 10}+B_{\lambda 11} \hat{k}_{s} \\
B_{\lambda 2}=B_{\lambda 20}+B_{\lambda 21} \hat{k}_{s}
\end{gathered}
$$

The mean-vortex-wavelength equation, given by Eq. (33), depends on the five coefficients $A \lambda_{0}, A \lambda_{1}, B \lambda_{0}, B \lambda_{1}$, and $B \lambda_{2}$, which are obtained from Eqs. (36)-(52).

Computational results obtained from this algebraic relation agree with Nikuradse's experimental data on flow in artificially roughened pipes ${ }^{6}$ as well as more recent experimental data presented by Shockling, Allen, and Smits. ${ }^{13}$ For example, Fig. 5 shows a comparison between experimental data for the Darcy friction factor ${ }^{6,13}$ and results obtained from the proposed algebraic relation. Notice that the current algebraic relation predicts a friction factor that becomes independent of Reynolds number as the Reynolds number becomes large. This is shown in Fig. 5 for bulk Reynolds numbers as large as $2 \times 10^{9}$.

Fully rough flow is defined to be the asymptotic high-Reynolds-number limit as the turbulent eddy viscosity $v_{t}$ becomes large compared to the molecular viscosity $v$ throughout the flow field. Because $v_{t}$ is smallest near the wall, the limit for application of the fully rough flow approximation can be evaluated by examining the near-wall behavior of $v / v_{t}$. For comparison, three curves of constant $\left(v / v_{t}\right)_{\text {wall }}$ are superimposed on the friction-factor results shown in Fig. 5. The dashed curve on the left is the locus of points having an eddy viscosity at the wall equal to the molecular viscosity. The roughness Reynolds number for all points along this curve is approximately 77 . Along the second dashed curve, the eddy viscosity at the wall is 10 times the molecular viscosity and the roughness Reynolds number is 766. The dashed curve on the right is the locus of points where the eddy viscosity at the wall is 100 times the molecular viscosity and the roughness Reynolds number is 7658. At a roughness Reynolds number of 1000, the molecular viscosity is nearly $8 \%$ of the eddy viscosity at the wall. For roughness Reynolds numbers below 1000 ,

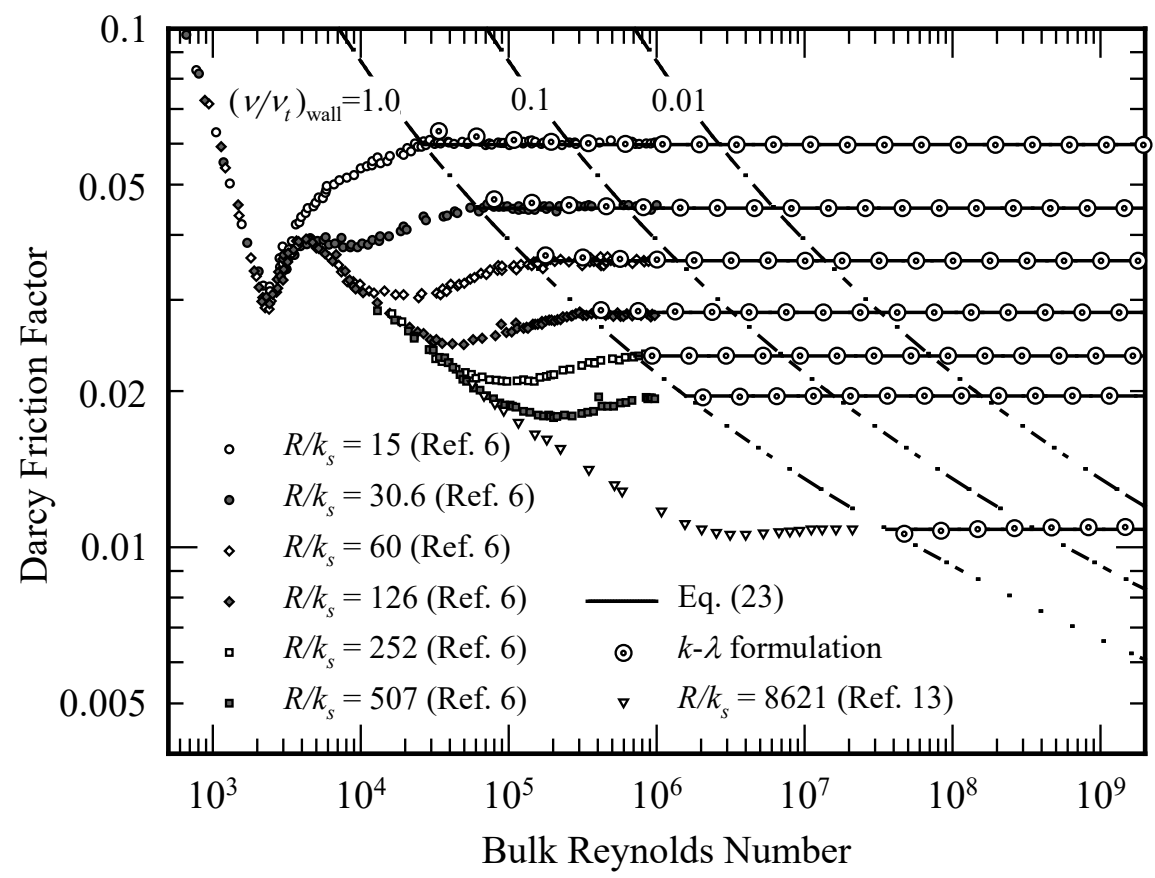

Figure 5. Darcy friction factor in rough pipes, predicted from the $k$ - $\lambda$ formulation with the algebraic relation for $\lambda$, using $\sigma_{k}=4.0$ and $k_{\mathrm{wal}}^{+}=0.1$. 
the molecular viscosity becomes more significant, and the fully rough flow approximation breaks down near the pipe wall. For fully rough flow, the velocity profiles compare well with the log law given by Eq. (26), as shown in Figs. 6 and 7. Results similar to those shown in Figs. 5-7 are obtained for any value of $\sigma_{k}$ in the range 2.0 to 6.0 and any value of $k_{\mathrm{wal}}^{+}$in the range 0.05 to 1.0 , provided that the relations given by Eqs. (35)-(52) are maintained.

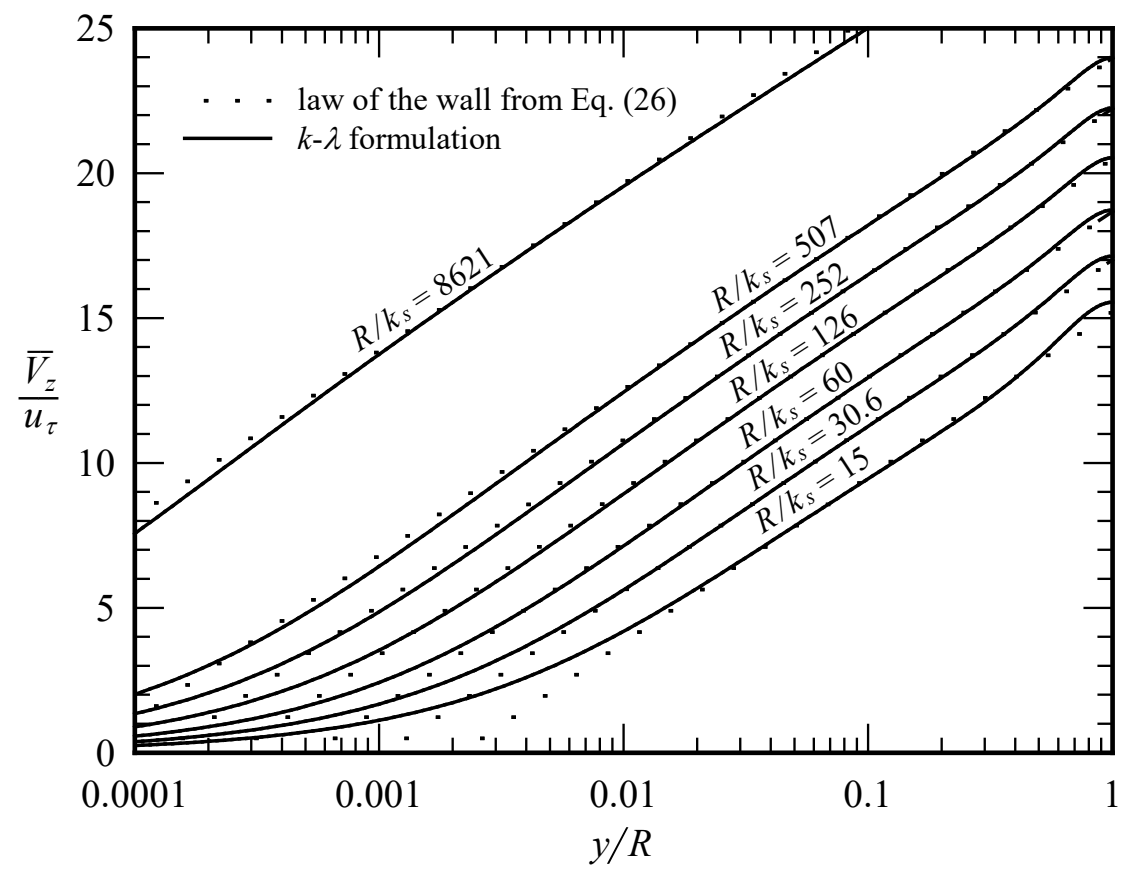

Figure 6. Velocity profiles in fully rough pipe flow, predicted from the $k-\lambda$ formulation with the algebraic relation for $\lambda$, using $\sigma_{k}=4.0$ and $k_{\mathrm{w} a l}^{+}=0.1$.

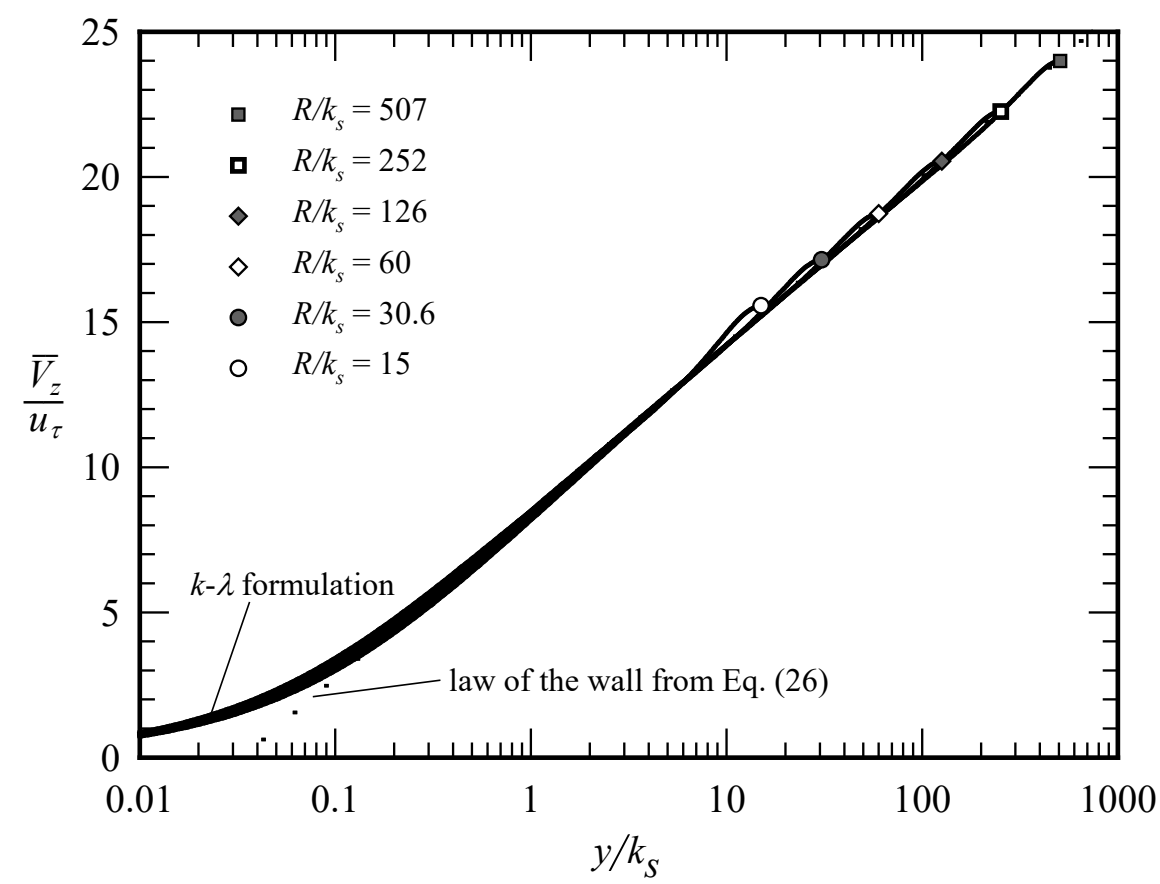

Figure 7. Velocity profiles in rough pipes at a roughness Reynolds number of 1000, predicted from the $k$ - $\lambda$ formulation with the algebraic relation for $\lambda$, using $\sigma_{k}=4.0$ and $k_{\mathrm{wal}}^{+}=0.1$. 
Because results obtained from Eqs. (19) and (20) combined with Eqs. (32)-(52) agree well with experimental data obtained for the velocity profile and friction factor at high Reynolds numbers, they can be used to predict the distributions of the turbulence variables in fully rough pipe flow. Example profiles for the turbulent kinetic energy, mean vortex wavelength, kinematic eddy viscosity, and RMS fluctuating vorticity are presented in Figs. 8-11, respectively.

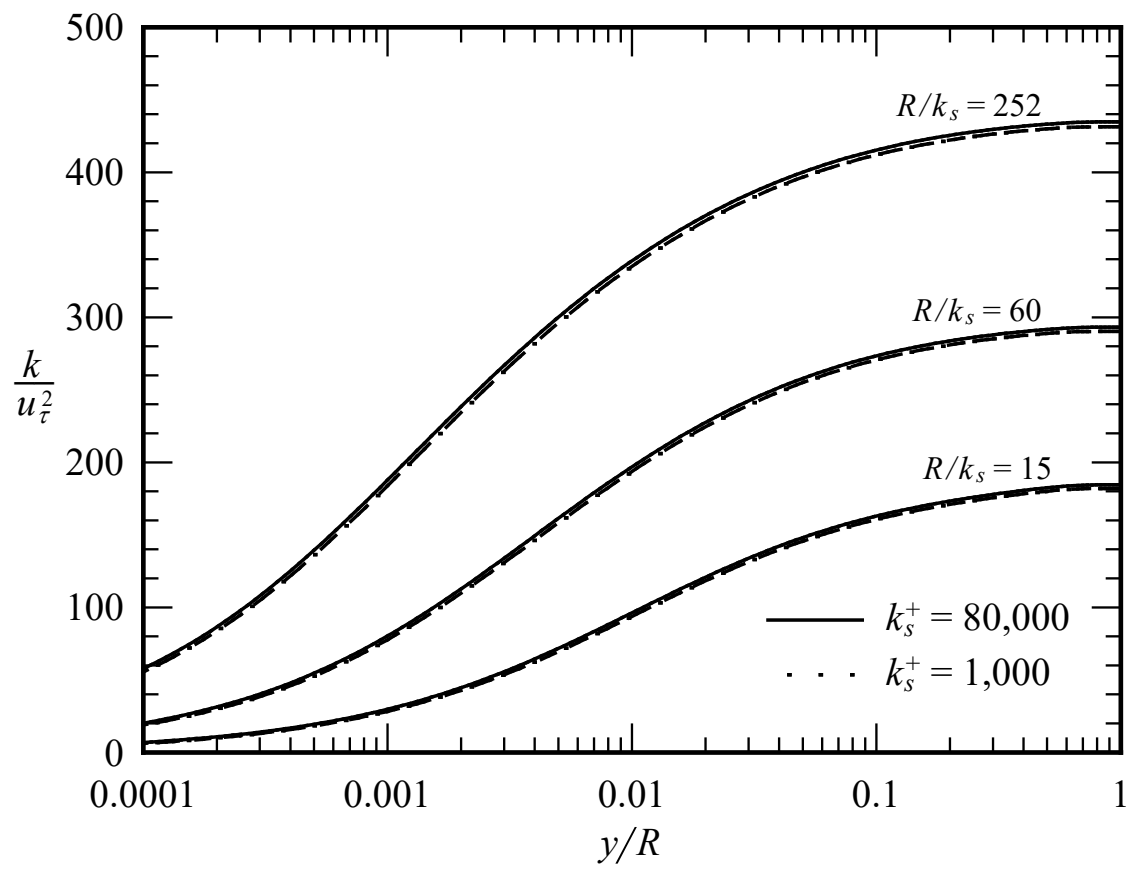

Figure 8. Turbulent-kinetic-energy profiles, predicted from the $k$ - $\lambda$ formulation with the algebraic relation for $\lambda$, using $\sigma_{k}=4.0$ and $k_{\mathrm{wal}}^{+}=0.1$.

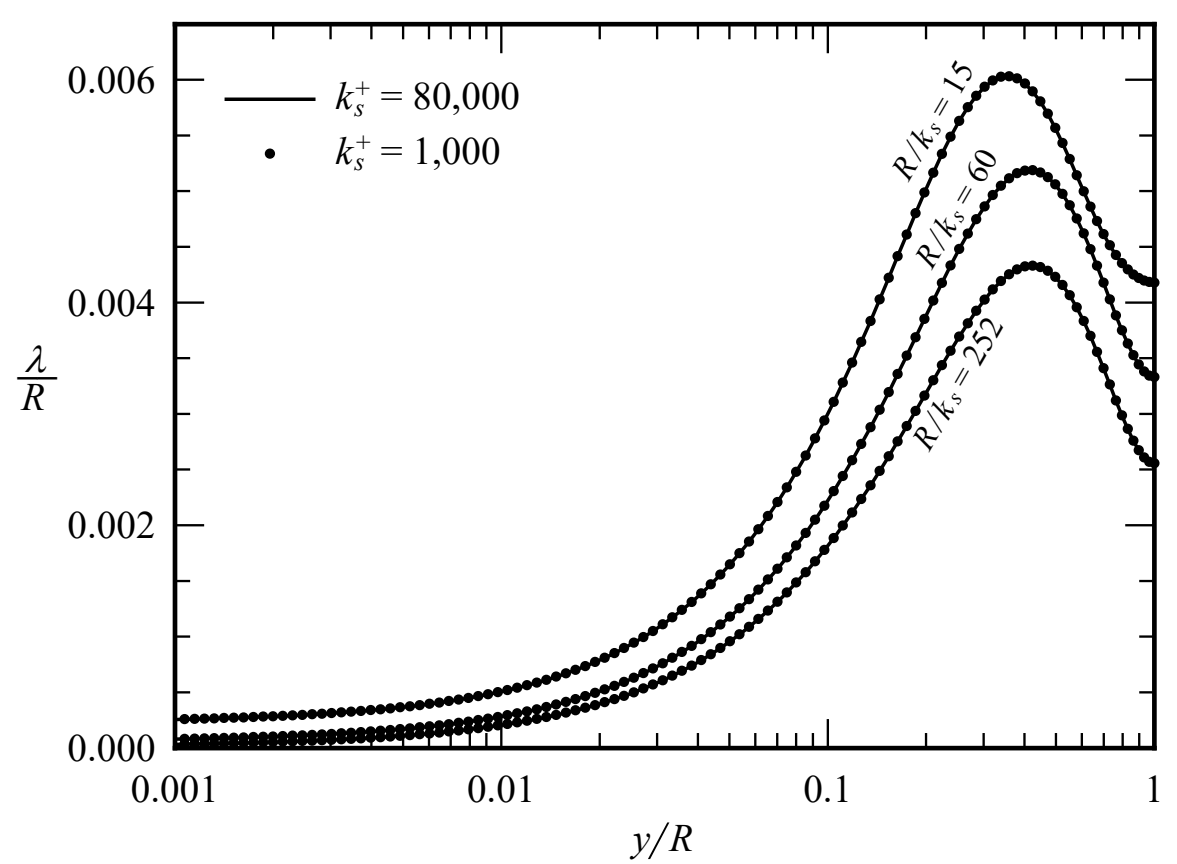

Figure 9. Mean-vortex-wavelength profiles, predicted from the $k$ - $\lambda$ formulation with the algebraic relation for $\lambda$, using $\sigma_{k}=4.0$ and $k_{\mathrm{w} a l}^{+}=0.1$. 


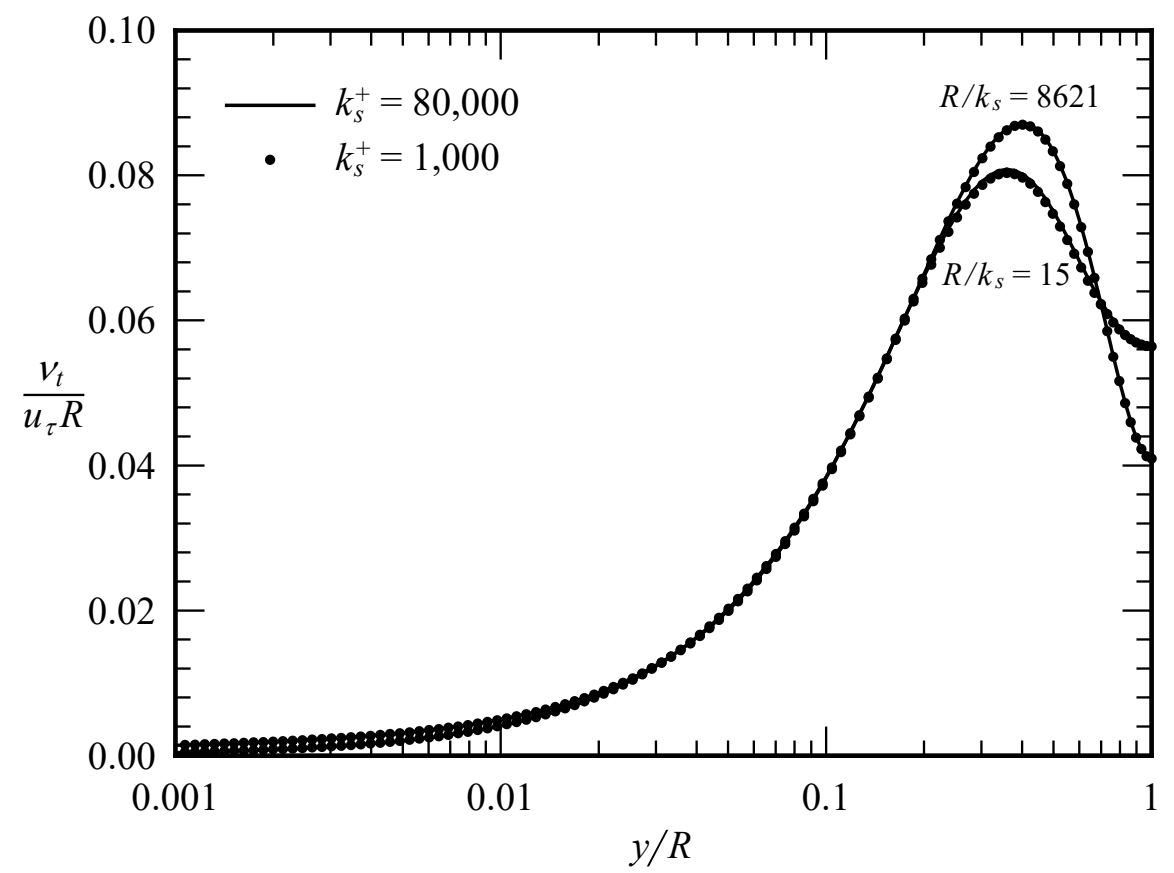

Figure 10. Turbulent-eddy-viscosity profiles, predicted from the $k$ - $\lambda$ formulation with the algebraic relation for $\lambda$, using $\sigma_{k}=4.0$ and $k_{\mathrm{wal}}^{+}=0.1$.

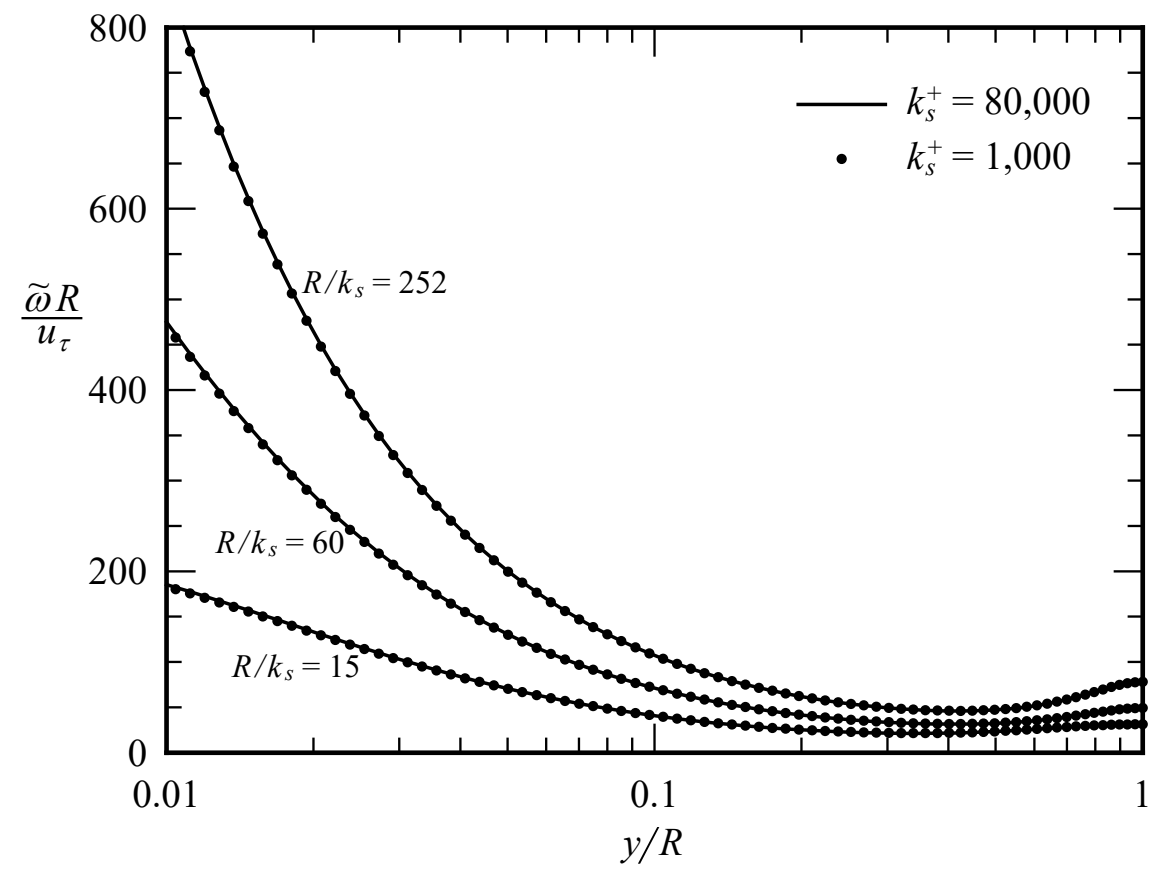

Figure 11. RMS-fluctuating-vorticity profiles, predicted from the $k$ - $\lambda$ formulation with the algebraic relation for $\lambda$, using $\sigma_{k}=4.0$ and $k_{\mathrm{wal}}^{+}=0.1$.

Notice from Figs. 8-11 that very slight variations in the turbulence variable distributions can be seen between the roughness Reynolds numbers of 1,000 and 80,000 . This is because, at these high roughness Reynolds numbers, the molecular viscosity is negligible when compared to the turbulent eddy viscosity throughout the flow field from the pipe centerline to the wall. 


\section{Conclusions}

For the case of incompressible flow, Eqs. (19) and (20) provide the foundation for an energy-vorticity RANS turbulence model that differs significantly from traditional energy-dissipation models, which include the established $k-\varepsilon, k-\omega$, and $k-\zeta$ models commonly used for CFD. Following an analogy with the kinetic theory of gases, the development of Eqs. (19) and (20) is based on a more direct analogy between turbulent and molecular transport.

The turbulent-energy-transport equation that is given in Eq. (20) contains two unknown closure coefficients; the viscous-dissipation coefficient, $C_{\lambda}$, and the turbulent-transport coefficient, $\sigma_{k}$. These coefficients should both be dimensionless universal constants. It has been shown here that excellent agreement with experimental data for velocity profiles and friction factors in fully rough pipe flow can be attained over the range of about $2<\sigma_{k}<6$ and $0.00001<C_{\lambda}<0.00056$, provided that the relation between $C_{\lambda}$ and $\sigma_{k}$ that is given in Eq. (35) is maintained.

In addition, the turbulent-energy transport equation requires a wall boundary condition for the specific turbulent kinetic energy, $k$. The specific turbulent kinetic energy at a rough surface should be proportional to the square of the friction velocity, $u_{\tau}$, as shown in Eq. (32). For incompressible flow, the dimensionless proportionality coefficient, $k_{\mathrm{wal}}^{+}$, is expected to be a unique function of the roughness Reynolds number, $k_{s} u_{\tau} / v$. As the roughness Reynolds number approaches zero, $k_{\mathrm{wal}}^{+}$should approach zero as well. By definition, fully rough flow occurs when the roughness Reynolds number is high enough so that the solution becomes independent of molecular viscosity. Hence, for fully rough flow, the dimensionless parameter, $k_{\mathrm{w} \mathrm{al}}^{+}$, must approach another universal constant associated with the turbulence model. It has been shown here that excellent agreement with experimental data for fully rough pipe flow can be attained over the range of about $0.05<k_{\mathrm{wal}}^{+}<1.0$, provided that certain relations are maintained between the mean vortex wavelength, $\lambda$, and the dimensionless parameter, $k_{\mathrm{wal}}^{+}$. Additionally, results show that the formulation can be used to predict the Darcy friction factor for fully rough pipe flow down to a roughness Reynolds number as low as about 100 , which is much lower than the ratio of molecular viscosity to eddy viscosity at the wall would imply.

It is important to recognize from the discussion above that excellent agreement with experimental data for fully rough pipe flow can be attained over a range of the model constants, which include $C_{\lambda}, \sigma_{k}$, and the fully rough limit

for $k_{\mathrm{wa}}^{+}$. In terms of future development, this is fortunate, because it provides a great deal of flexibility that can be used when tuning the model to agree with experimental data for other turbulent flows.

\section{References}

${ }^{1}$ Boussinesq, J., "Essai sur la Théorie des Eaux Courantes," Mémoires Présentés par Divers Savants à l'Académie des Sciences de l'Institut de France, Vol. 23, No. 1, 1877, pp. 1-680 (Presented Oct., 1872).

${ }^{2}$ Phillips, W. F., "Foundation for an Energy-Vorticity Turbulence Model," Presented, 42nd AIAA Fluid Dynamics Conference, New Orleans, Louisiana, 25-28 June 2012.

${ }^{3}$ Robinson, D. E., Harris, J. E., and Hassan, H. A., "Unified Turbulence Closure Model for Axisymmetric and Planar Free Shear Flows," AIAA Journal, Vol. 33, No. 12, 1995, pp. 2325-2331.

${ }^{4}$ Robinson, D. E., and Hassan, H. A., "Further Development of the $k-\zeta$ (Enstrophy) Turbulence Closure Model," $A I A A$ Journal, Vol. 36, No. 10, 1998, pp. 1825-1833.

${ }^{5}$ Kreuzinger, J., Friedrich, R., and Gatski, T. B., "Compressibility Effects in the Solenoidal Dissipation Rate Equation: A Priori Assessment and Modeling," International Journal of Heat and Fluid Flow, Vol. 27, 2006, pp. 696-706.

${ }^{6}$ Nikuradse, J., "Strömungsgesetze in Rauhen Rohren," VDI Forschungsheft, Vol. 361, 1933, pp. 1-22.

${ }^{7}$ Colebrook, C. F., "Turbulent Flow in Pipes, with particular reference to the Transition Region between the Smooth and Rough Pipe Laws," Journal of the Institution of Civil Engineers, Vol. 11, 1939, pp. 133-156.

${ }^{8}$ Moody, L. F., "Friction Factors for Pipe Flow," Transactions of the A.S.M.E., Vol. 66, 1944, pp. 671-684.

${ }^{9}$ Schlichting, H., "Rough Pipes and Equivalent Sand Roughness," Boundary Layer Theory, Fourth Edition, McGraw-Hill, New York, 1960, pp. 519-527.

${ }^{10}$ Schlichting, H., "Experimentelle Untersuchungen zum Rauhigkeitsproblem," Ingenieur-Archiv, Vol. 7, No. 1, 1936, pp. 1-34.

${ }^{11}$ Wilcox, D. C., "Surface Roughness" and "Companion Software: Channel and Pipe Flow," Turbulence Modeling for CFD, 2nd ed., DCW Industries, Inc., La Cañada CA, 1998, pp. 175-177 and 433-439.

${ }^{12}$ Wilcox, D. C., "Surface Roughness" and "Companion Software: Channel and Pipe Flow," Turbulence Modeling for CFD, 3rd ed., DCW Industries, Inc., La Cañada CA, 2006, pp. 182-185 and 477-478.

${ }^{13}$ Shockling, M. A., Allen, J. J., and Smits, A. J., "Roughness Effects in Turbulent Pipe Flow," Journal of Fluid Mechanics, Vol. 564, 2006, pp. 267-285.

${ }^{14}$ Richardson, L. F., and Gaunt, J. A., "The Deferred Approach to the Limit," Transactions of the Royal Society of London, A, Vol. 226, Jan. 1927, pp. 299-361.

${ }^{15}$ Fowler, E. B., "Evaluation of Turbulence Variable Distributions for Fully Rough Pipe Flow", Ph. D. Dissertation, Department of Mechanical and Aerospace Engineering, Utah State University, Logan, UT, 2012. 
${ }^{16}$ Hunsaker, D. F., "Evaluation of an Incompressible Energy-Vorticity Turbulence Model for Fully Rough Pipe Flow”, Ph. D. Dissertation, Department of Mechanical and Aerospace Engineering, Utah State University, Logan, UT, 2011.

${ }^{17}$ Broyden, C. G., "The Convergence of a Class of Double-rank Minimization Algorithms," Journal of the Institute of Mathematics and Its Applications, Vol. 6, 1970, pp. 76-90.

${ }^{18}$ Fletcher, R., "A New Approach to Variable Metric Algorithms," The Computer Journal, Vol. 13, 1970, pp. 317-322.

${ }^{19}$ Goldfarb, D., "A Family of Variable Metric Methods Derived by Variational Means," Mathematics of Computation, Vol. 24, 1970, pp. 23-16.

${ }^{20}$ Shanno, D. F., "Conditioning of Quasi-Newton Methods for Function Minimization," Mathematics of Computation, Vol. 24, 1970, pp. 647-656. 